\title{
SOBRE LA TRANSMISION TEXTUAL DE LONGO
}

Longus's text has been transmitted to us in three manuscript families. One of them, the Ursinian, was considered as formed by both Vaticani graeci 1347 and 1350, and Parisinus graecus 2895. Tubingensis $\mathrm{Mb}$ I6 was considered among the apographi and directly dependant on Vat. gr. 1347. This paper sustains on the contrary that Tubingensis $\mathrm{Mb} \mathrm{I} 6$ is not only the common origin of both Vaticani above mentioned - as it had already been pointed out by Vilborg in his edition of Achilles Tatius - but also the source of Parisinus graecus 2895 .

Longo nos ha sido transmitido por tres familias de códices, designadas por Dörrie ${ }^{1}$ como Florentina, Vaticana y Ursiniana.

La Florentina la compondrian el Laurentianus conv. sopp. 627 de fines del xIII o comienzos del xIv (sigla A en Dörrie) y un manuscrito perdido del que se nos ha transmitido muchas de sus lecciones en anotaciones marginales del Vaticanus Gr. I347, lo que Dörrie simboliza por $\wedge$.

La Vaticana, formada por el Vaticanus Gr. I348, de finales del xIV según Dörrie (que lo designa por $Q$ ), de comienzos del xvI en opinión de $\mathrm{H}$. van Thiel ${ }^{2}$ o de mediados del $\mathrm{xvI}$ en la de E. Vilborg ${ }^{3}$.

Por último, la Ursiniana, que es el objeto especial de nuestro estudio, estaría formada por tres manuscritos básicos:

N Parisinus Gr. 2895, escrito poco antes del 1529 , ya que en ese año Jerónimo Fondulo a petición de Francisco I lo envió a Paris ‘. Por las marcas de agua el papel es del segundo cuarto del siglo xvi, según nos informa Dörrie (op. cit., p. rI).

1 En su tesis De Longi Achillis Tatii Heliodori memoria, Gotinga, 1935, p. 43 y 89 .

-Ueber die Textüberlieferung des Longuss, $R h . M$. 104, 1961, p. $35^{8}$.

- En su edición de Leucipe y Clitofón de Aquiles Tacio, Estocolmo, 1955. p. XXVI.

- Vid. Dörrie, op. cit., p. II. 
U Vaticanus Gr. 1347, copiado, según nos dice el autor mismo, Fulvio Orsini, al final del texto de Longo, el año I553. De éste, siempre según Dörrie, depende el Tubigensis $\mathrm{Mb} \mathrm{I6}$, copiado por Juan Honorio Hydruntino, de quien no tenemos noticias posteriores a $1565^{1}$. Este manuscrito es fechado por Dörrie (op. cit., p. I6) "paulo post annum I553». El códice vaticano fue corregido por el mismo Orsini con conjeturas suyas o con lecciones de otro códice desconocido, que añadió al margen, lo que como dijimos anteriormente Dörrie simboliza por V.

Y Vaticanus Gr. I350, del mismo autor que el Tubingensis y dependiente del mismo manuscrito que $U$, a la vez que enriquecido con las notas y adiciones del último, aunque posteriormente fueron borradas.

Por tanto, el stemma de Dörrie para la familia Ursiniana, a la que por ahora nos limitaremos, es el siguiente:



Veinte años después, E. Vilborg ${ }^{2}$ demuestra para el texto de Aquiles Tacio que el manuscrito Tubingensis $\mathrm{Mb} \mathrm{I} 6$, al que da la sigla $\mathrm{T}$, que nosotros usaremos en adelante, no es descendiente del Vat. Gr. $1347(\mathrm{U})$, sino que éste es apógrafo de aquél. A su vez, del U depende el $\mathrm{Y}$ (Vat. Gr. 1350) en lo que se refiere a Longo completo y los diez primeros capítulos de Aquiles Tacio, único fragmento que conservan los Ursinianos de este novelista.

E1 stemma, entonces, para la familia que nos ocupa, sería según Vilborg:



En lo anterior $\xi$ representa "consensus codicum NT" (op. cit., p. LXVI). Se ha de observar también que la alteración por Vilborg del stemma

1 Vid. Vogel M. Gardthausen, V., Die griechischen Schreiber des Mittelalters und der Renaissance, XXXIII Beiheft zum Zentralblatt für Bibliothekswesen, Leipzig, 1909, p. 183 .

" Vid. Vilborg, op. cit., pp. LX, LXIV-LXVI y LXXVI. 
de Dörrie, no representa modificación de las fechas de los manuscritos implicados, ya que para T sigue dando la referencia de Dörrie al I553, como indica el que utilice este año para "post quem" de un apógrafo del Tubingensis, el Paris. Gr. 2903 (op. cit., p. XXI), lo que le lleva a la contradicción de aceptar que el Vat. Gr. 1347 fue copiado después del Par. Gr. 2903, ya que éste fue copiado antes de enmendar los errores, mientras el Vat. Gr. I347 lo fue después de enmendar los errores de $T$, según nos dice Vilborg en la p. LXVI. La consecuencia lógica sería adelantar la fecha tanto del Tubingensis como del Parisino.

Un detenido estudio de la familia Ursiniana nos confirma la suposición anterior.

Al tener que reexaminar la tradición manuscrita de Longo, con vistas a una edición que sobre este autor preparamos, observamos que el stemma de Vilborg era sólo parcialmente exacto y exigfa nuevas precisiones, ya que como probaremos a continuación el Tubingensis $\mathrm{Mb}$ I6 es el manuscrito del que procede inmediatamente el Parisinus Gr. $2895(\mathrm{~N})$, y nuestro stemma sería por tanto:



Errores comunes N' ${ }^{1}$

242.I8 $8 \varepsilon \varphi \cup \lambda \propto \gamma \mu \varepsilon v \omega s$ AQUY

$244.23 \tau \varepsilon$ 華 $\mathrm{AQ}$

$258.5 \mu \alpha k p d v$ AQUY

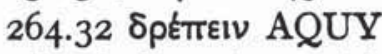

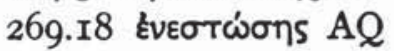

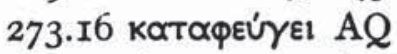

282.32 өєрно́тєро AQUY

292.I7 हүкрú $\psi \alpha \sigma \alpha A Q$

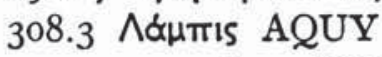

323.22 крณтก̃ $\alpha$ AQUY

$315.7 \pi \rho \circ \alpha \lambda \varepsilon \sigma \alpha \mu \varepsilon v 05$ AQUY

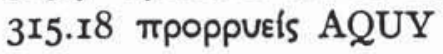

$\pi \varepsilon \phi \cup \lambda \propto \gamma \mu \dot{v} \nu \eta \nu \omega s$ TN

ฤ๐ TN oũv UY

Hokpd TN

SpÉtrteıv TN



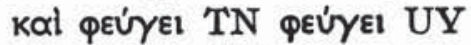

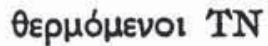

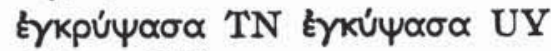

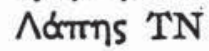



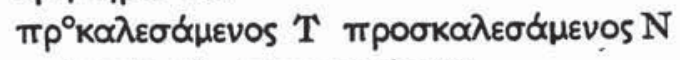
$\pi \rho^{\circ}$ pueís $\mathrm{T}$ mpoopueis $\mathrm{N}$

1 Las siglas de los manuscritos serán las anteriormente utilizadas de Dörrie salvo para el Tubingensis Mb I6, que será la usada por Vilborg, $T$, para evitar la posible confusión $U / \mathbf{u}$. Las cifras envian a las páginas y lineas de $\mathbf{R}$. Hercher, Erotici Scriptores Graeci, Leipzig, 1858 . 
La lista podría ampliarse, aunque a nuestro entender son más probatorios de la dependencia algunos errores exclusivos de $\mathrm{N}$ motivados ya por una mala lectura en $T$, o, lo que es más frecuente, una deficiente interpretación de las abreviaturas de T. En este apartado se deben incluir también los errores corregidos de $\mathrm{N}$, pero que indican que la lectura errónea de $\mathrm{N}$ tiene su origen en $\mathrm{T}$.

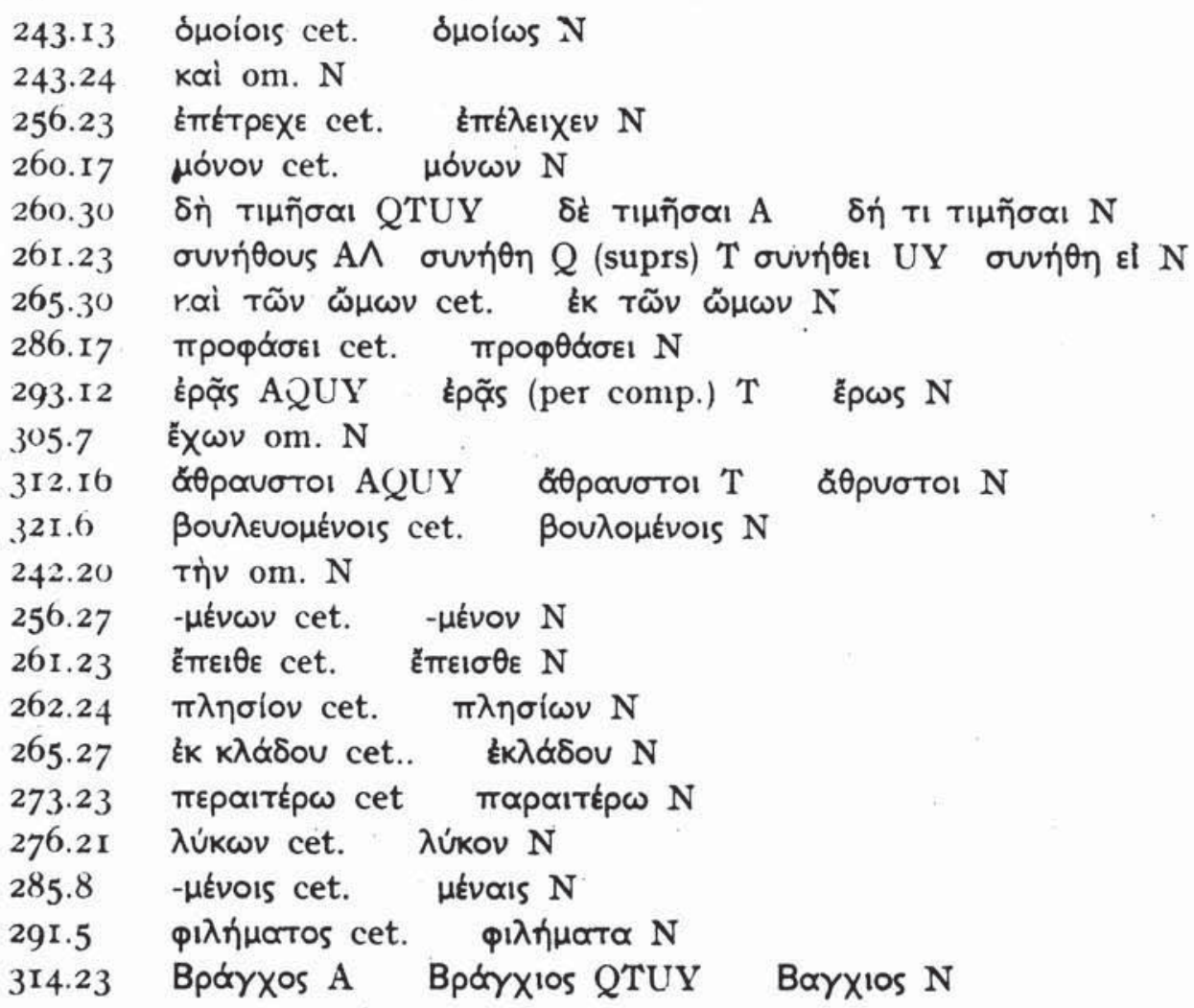

Hay algunos otros casos que merecen una explicación más detallada: En 305.23 $\mathrm{N}$ añade al margen elvaı para sustituir a un kal tachado. $\mathrm{L}$ a confusión de $\mathrm{N}$ está claramente motivada por la presencia en $\mathrm{T}$



En $245.4 \mathrm{~N}$ tacha ĩ $\sigma \omega s$ tras ő $v \propto \rho$. Cómo se ha podido introducir I $\sigma \omega$ s en el texto de $\mathrm{N}$ se explica al ver la secuencia textual de $\mathrm{T}$. En este manuscrito la línea acaba en o $v \propto \rho$ y a la misma altura, a continuación, hay un lows correspondiente a una conjetura que hace el copista a la línea inmediatamente inferior.

En 307.30 y $312.14 \mathrm{~N}$ añade al margen las líneas omitidas, líneas que precisamente coinciden con homoioteleuta en $\mathrm{T}$. 
En $326.5 \mathrm{~N}$ supraescribe la desinencia - $\mu \varepsilon v \propto$ a la errónea - $\mu \propto \rho$. Si comprobamos el texto de $T$ veremos que es fácil confundir la abreviatura para la desinencia del participio con $-\mu \propto \rho$.

Los numerosísimos casos de itacismo que ofrece $\mathrm{N}$ pueden tener también una explicación no fonética, sino de escritura. Así, los frecuentes casos de $T \eta$ S por TIS pueden tener su origen en la similitud de las abreviaturas ${ }_{\tau}^{\hat{s}} \cdot s_{\tau}^{*}$, respectivamente.

La similitud entre $-\tau$ y $-\tau \alpha$ ( $\tau$ y $\tau$ :, respectivamente) es causante de algunos errores introducidos en la familia Ursiniana, como es el caso de 3r3.3r:

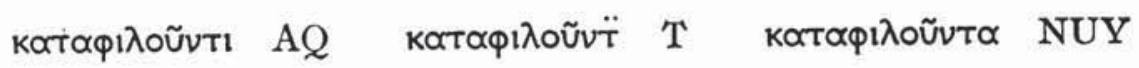

Otros errores de la Ursiniana pueden ser rastreados también en el Tubingensis, como es el caso, para citar algún ejemplo, de 254.r4:

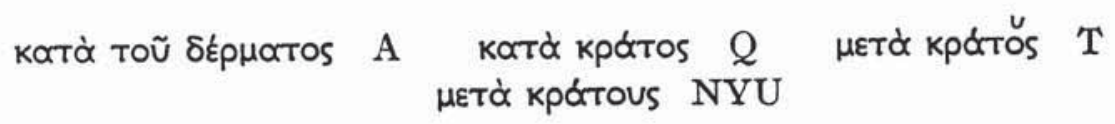

donde $\mathrm{T}$ confundió primero las abreviaturas de las preposiciones koт́k y $\mu \varepsilon \tau\{\alpha$, y posteriormente corrigió el caso para ponerlo de acuerdo con la preposición.

Un caso similar, donde una mala lectura hecha por $\mathrm{T}$ y después corregida da lugar a una nueva variante, es 269.5:

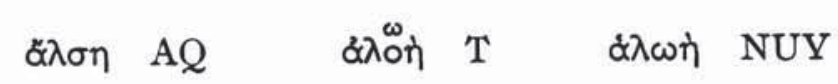

Es manifiesto que $\mathrm{T}$ confundió la sigma con la ómicron y posteriormente corrigió lo que consideraría un lapsus fonético.

Creemos que las pruebas aportadas son suficientes para demostrar la dependencia del Parisinus Gr. $2895(\mathrm{~N})$ respecto del Tubingensis $\mathrm{Mb}$ I6, lo que, unido a lo ya expuesto por Vilborg, nos lleva a afirmar que el único representante válido, si exceptuamos los anotaciones marginales del Vat. Gr. I347, de la familia Ursiniana es el mencionado Tubingensis, ya que todos los demás resultarían ser apógrafos de él.

Francisco Romero 